\title{
Filigrane
}

Écoutes psychanalytiques

\section{Le sujet de la violence : objectalisation structurante ou objectivation déshumanisante?}

\section{Sophie Gilbert}

Volume 25, numéro 1, printemps 2016

Actes du colloque Le sujet sacrifié (Ghyslain Lévy)

URI : https://id.erudit.org/iderudit/1037369ar

DOI : https://doi.org/10.7202/1037369ar

Aller au sommaire du numéro

Éditeur(s)

Revue Santé mentale au Québec

ISSN

1192-1412 (imprimé)

1911-4656 (numérique)

Découvrir la revue

Citer cet article

Gilbert, S. (2016). Le sujet de la violence : objectalisation structurante ou objectivation déshumanisante ? Filigrane, 25(1), 11-18.

https://doi.org/10.7202/1037369ar d'utilisation que vous pouvez consulter en ligne. 


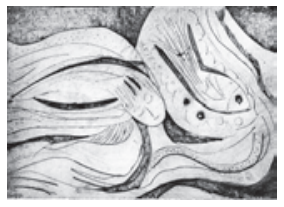

\title{
Le sujet de la violence: objectalisation structurante ou objectivation déshumanisante?
}

\author{
Sophie Gilbert
}

2016 . La violence est partout. Dans les médias, les images se font en direct, et pas les moindres. La plus vile cruauté nous est montrée sans voile, sans pudeur. L'humain, la victime comme le bourreau, n'a plus besoin de masque: celui qui tue le fait sans gêne, celui qui est sauvagement abattu nous montre sa détresse, son désespoir, les restes de son humanitude (Paillé, 2006) avant de perdre définitivement son statut de vivant... Car celui de sujet, il l'avait déjà sacrifié, du moins, dans les yeux de son agresseur et possiblement, de son public. Puis, on passe à autre chose, on vaque à ses occupations, on continue... à vivre?

La violence est partout. Les jeunes, les enfants même, y sont surexposés. Et pas seulement dans les pays en guerre: si on ne la vit pas, il faut y jouer. Dans des décors d'un réalisme désarmant, nos jeunes, manettes en mains, prétendent être des soldats; ils défendent leur pays et envahissent celui des autres, les étrangers. Le nouveau visage de la colonisation? La vie serait-elle un éternel recommencement?

C'est aussi l'ère du déni. Comment y échapper? Sinon, pas de vie possible, me direz-vous. Dans l'état actuel du monde, on ne peut sans culpabilité, sans honte, investir son travail, nourrir ses enfants, etc. Il faut poser une limite, une limite interne (à défaut de pouvoir même envisager de s'opposer explicitement et efficacement, de l'extérieur), à la violence ambiante. Il faut la nier, l'oublier... et pourtant: elle nous revient toujours, le lendemain généralement, sous une autre forme: inusitée, plus provocante encore, voire plus «fascinante»?

Le clinicien d'aujourd'hui n'échappe pas à cette réalité. Si la violence franchit la porte de son cabinet, le déni risque aussi de s'y immiscer. Lorsqu'un psy refuse de "prendre tel cas» parce que trop "agissant», 
lorsqu'il ne peut prendre le risque de se confronter à la «folie» adolescente, aux agirs, aux tentatives de suicide répétées, à l'automutilation ou aux scarifications. S'agit-il d'un espace clinique aseptisé? Il semble que déjà, cet espace " propre $^{1}$ » que l'on peut retrouver autant en psychanalyse que dans d'autres univers psychothérapiques dévoile une vision de l'autre objectivé; toute trace de subjectivité et de singularité étant ignorée dès lors que seul un symptôme est considéré (par le choix arbitraire d'un parmi les autres) avant que ne tombe le verdict: "pas dans ma cour». N'est-ce pas justement par ce mécanisme d'objectivation de l'autre que les pires dictatures ont pu naître et demeurer quasi inaltérées jusqu'à ce jour? Que les pires cruautés peuvent être mises en œuvre puis données à voir — pour ne pas dire: être délibérément regardées? Que penser de cette violence de la réduction de l'autre à son symptôme? S'agit-il d'une utilisation détournée (pervertie?) du trait unaire (Lacan), de l'altérité pure - est-ce que les tragiques impacts sociaux de cette vision, qui a soutenu plus d'un génocide, pourraient prendre racine à l'échelle individuelle, dans un simple regard posé sur l'autre? Ou encore, peut-on déceler dans cette exclusion sans appel des traces de la violence fondamentale (Bergeret), en ce que le clinicien se sent tenu de rejeter l'autre, le patient, pour protéger son intégrité?

Si la violence inhérente à certaines réactions des psys est ici questionnée, reste que la recrudescence actuelle de cette violence qui leur est imposée, ou plutôt « donnée à voir » — par diverses mises en scène impliquant le corps demeure inexpliquée. À première vue, dans ces diverses formes d'agirs sur le corps et par le corps (coupure chez l'un, injection chez l'autre, mais aussi maigreur ultime, et assaut cruel d'autrui), difficile de retrouver des traces de la «belle indifférence» hystérique. L'atteinte au corps hystérique, signifiante et en attente de traduction, fait place aujourd'hui à une violence, qui davantage qu'un quelconque message crypté, envahit cette «adresse» au psy trop souvent confiné à une position de spectateur impuissant. Cela pourrait d'ailleurs justifier la réserve - voire même, l'attitude défensive - de certains cliniciens: le pressentiment que quelque chose d'intolérable leur sera imposé, voilé sous la réassurance de l'attribution de ce malaise à un symptôme circonscrit, nommable, objectivable, et par là même, facile à exclure de sa pratique.

À l'échelle sociétale, cette forme actuelle de la violence imposée à l'autre en passant par la force de l'image surtout (associée à un message verbal stéréotypé ou carrément absent ${ }^{2}$ ) met essentiellement en scène un «corps»... d'abord vivant, puis violenté, qu'on ne peut regarder qu'en 
faisant abstraction de l'humanité qui lui est pourtant associée. Seul remède au tragique de cette vision d'horreur? Dénaturer l'humain, comme si on le «tuait» avant même d'assister à son dernier souffle. Si de telles coutumes ont pu être acceptées à des époques antérieures — pensons à l'attraction publique que ces scènes pouvaient susciter à l'ère médiévale — il est difficile de les dissocier d'une vision régressive de l'humain. Rapidement, on pourra se dire que cette violence est celle du «barbare ${ }^{3} »$, de l'étranger.

Néanmoins, la réalité de ce que voit — ou refuse parfois de voir? — le clinicien dans son cabinet, dans les médias, et même dans la culture démontre qu'il est douteux de considérer que seuls les autres, les étrangers - j'entends ici les non-occidentaux - feraient encore preuve aujourd'hui de cette forme de violence. En effet, dès lors que l'on considère par exemple le rapport au corps inhérent à celle-ci, ne reconnaît-on pas les fondements d'une attraction publique — différente des décapitations, je vous l'accorde — fort «glamour» dans l'exposition «Bodies» qui a fait le tour du monde? N'est-ce pas ici une preuve de la facilité avec laquelle l'humain-spectateur peut se dissocier de son identification (nécessaire) à l'autre? Et curieusement, n'estce pas dans cette exacte situation, où la psyché est expulsée du corps-mort, que se produit de façon saisissante une identification massive à l'autre? En effet, privé de son humanité, de son empathie, de cette reconnaissance de l'autre comme similaire à soi, de la prise en compte du «sacré», que restet-il du sujet humain dans ce regard porté sur le corps-objet? La violence de la réification de l'autre est-elle indissociable de la propre objectivation du sujet-témoin? Le voyeur ne trouve-t-il pas une certaine jouissance dans l'identification au bourreau? Et la violence reléguée à l'autre, au bourreau, n'est-elle pas tout d'abord la violence de soi que l'on refuse d'admettre — la projection étant l'échappatoire humaine de prédilection?

Ce détour par les enjeux macrosystémiques nous semble propice à reconsidérer la façon dont les cliniciens peuvent se retrouver témoins de la violence de l'autre, mais aussi, faisant violence à l'autre par des processus similaires à ceux relevés à l'échelle sociétale : le déni (ça ne me concerne pas), l'objectivation de l'autre, la désidentification (de l'autre singulier, ou d'une culture en particulier) - qui pourraient voiler en fait une suridentification à l'autre (à la violence du bourreau, au statut d'objet de la victime).

Si les psychanalystes et les cliniciens d'orientation psychanalytique s'entendent aisément sur la violence inhérente à certaines approches — par symptômes, par diagnostic DSM, par les «techniques d'intervention », par le testing — de cliniciens d'autres allégeances théoriques, il serait parfois 
plus difficile de prendre en considération, avec recul, les failles possibles de la référence théorico-clinique qui est la nôtre ${ }^{4}$. Pourtant...

- Au-delà de l'universalité des processus psychiques fondamentalement humains que nous venons de répertorier, le psychanalyste ou le clinicien d'orientation psychanalytique n'aurait-il pas lui aussi la tentation de comprendre à tout prix, de diagnostiquer, d'imposer ses vues sur l'autre?

- Nous l'avons vu, le psy n'est pas à l'abri, dans son cabinet, de l'attitude couramment adoptée devant l'écran (de télé, d'ordinateur); devant l'horreur, l'impensable, le "trop" (et le risque d'une identification massive), la tentation est forte d'objectiver l'autre, de le priver de ses qualités de sujet et même de son humanité, tout en niant toute identification possible;

- Faire violence en imposant une seule façon de faire, le détour par la parole... est-il nécessaire? Si cette parole s'avère impossible, ne serait-ce que dans un premier temps, qu'avons-nous à proposer? Que sommes-nous prêts à céder sur notre propre désir d'analyser par le médium du discours?

- Il est parfois trop facile d'oublier que la psyché est en fine articulation avec le corps... et ainsi, d'ignorer les messages adressés par ce corps (qui grossit ou à l'inverse, maigrit; ce corps tendu voire arqué; ce corps sans cesse en mouvement ou alors, complètement figé; ce corps séduisant ou repoussant, etc.). Les psychanalystes, spécialistes du développement et du fonctionnement (intra) psychiques ne sont pas à l'abri du refuge dans la psyché (dans un mécanisme au plus proche de l'intellectualisation), parfois plus confortable que la prise en compte de son corps et de celui de l'autre;

- La violence de l'interprétation (la violence secondaire, selon Aulagnier) demeure un risque de notre approche: ce discours, cette intervention ou interprétation, présentée comme une vérité, LA vérité... propice à rendre l'autre «fou»;

- L'Idéal — de la psychanalyse - peut aisément basculer dans un refuge idéologique (message stéréotypé par excellence): il faut être «analysé». Telle une religion, cet idéal ne saurait être remis en question et ce faisant, ouvre la voie aux pires abus sur l'autre qui se montre réticent... mais qui ose peut-être enfin "être lui-même»? La psychanalyse peut-elle à l'occasion constituer une idéologie où le clinicien refuse d'entrevoir un mieux-être parfois nécessaire, qui en passe par 
l'autre, voire même par une autre approche... fut-elle médicalisée? Ce qui sous-tend non seulement la question des visées (thérapeutiques ou non) de la psychanalyse, mais aussi celle de la collaboration avec d'autres professionnels. Y a-t-il là, en outre, un risque d'isoler le sujet pour son bien, de son propre environnement social, culturel?

- Et dans la même veine, combien de psys vont faire abstraction de la culture de l'autre sous prétexte d'universalité du fonctionnement psychique et de la métapsychologie, voire de la prépondérance de la réalité psychique sur la réalité socialement partagée... abrogeant du même souffle tout un pan de l'être, dont l'histoire, la préhistoire, les valeurs et la compréhension du monde telle que transmise par ses aïeux. Signe légitime du niveau — intrapsychique — d'une approche, ou réduction de la complexité du sujet?

$\mathrm{Du}$ reste, la psychanalyse donne aussi des lignes directrices, une compréhension du fonctionnement psychique qui peut permettre d'éviter de pervertir une relation thérapeutique où le sujet — dès lors considéré comme objet — s'estomperait au profit de la jouissance du clinicien. C'est là pour Lacan un constituant de l'éthique de la psychanalyse. Une éthique à différencier de la morale, nous dira Lacan: la place accordée au désir de l'analysant, au désir du sujet qui pourra bien sûr s'avérer en porte à faux avec celui du social, avec ce que l'autre veut pour lui, de lui. Sommes-nous toujours prêts à maintenir ce regard et cette écoute sur ceux qui nous consultent, ou fait-on parfois violence au sujet, sous prétexte de vouloir qu'il aille mieux? Le clinicien d'orientation psychanalytique saurait-il se distinguer à ce point de l'environnement socioculturel dans lequel il s'inscrit, mais aussi, dans lequel s'inscrivent ceux qui consultent et leurs proches (référents, parents, amis qui veulent leur bien, leur mieux-être)? Jusqu'où arrive-t-on à tenir cette posture éthique où le désir ne correspond pas toujours à la morale du «bien»?

D'autant plus que les cliniciens s'inscrivent dans un système de soins (qui veut justement le «bien», le mieux-être de la population), et si ce n'est le cadre institutionnel (pour ceux qui travaillent en cabinet privé uniquement), ce sera bien vite le cadre socioculturel, voire sociopolitique qui leur sera imposé ${ }^{5}$. N'est-ce pas ici une forme de violence subie par les psys que celle de l'obligation à la guérison rapide, tangible, mesurable? Comment les psys d'allégeance psychanalytique peuvent-ils rivaliser avec les philosophies actuelles de l'entreprenariat et de la rentabilité portées par toute une culture - particulièrement évidente, ces jours-ci, au Québec? Comment 
faire valoir la donne, incontournable pour nous, du temps? Car si la violence est partout, la temporalité n'est plus, la décharge est immédiate. Nous la rencontrons bien sûr dans la société éplorée devant tant d'agirs portés à l'écran en temps presque réel, mais aussi chez les individus, en particulier peut-être les jeunes. La décharge pressante sur le corps, par le corps, dans un refus de l'assujettissement à l'Autre (Lévy, 2013) sans prendre le risque de créer un espace, un temps d'élaboration nécessaire au travail psychique adolescent, à la mise en sens du pubertaire (Gutton). A-t-on le temps de rencontrer ces ados qui sortent de l'urgence, diagnostic ( TPL»: trouble de personnalité limite) et médication à la main après avoir attenté une énième fois à leurs jours?

Mais à l'inverse, jusqu'où peut-on tenir cette posture éthique psychanalytique tout en ne contrevenant pas au bien du sujet? Y a-t-il risque, à l'extrême, d'encourager malgré soi l'agir et l'échec de la sublimation, la décharge de tension pour soi peu importe le moyen, plutôt que dans le socialement acceptable où le désir serait d'emblée, en partie, contrecarré (voir le Malaise...) ? À l'inverse, le patient doit-il se «faire violence» pour aller mieux, de connivence avec certaines approches qui feraient aisément violence à l'autre pour son bien... considérant que la survie psychique serait similaire à celle du corps que l'on attaque par la médication, par la chirurgie (L'Archevêque et Bourgeois-Guérin, 2016)? Doit-on parfois l'amputer de son symptôme, de force? Ce faisant, on imagine bien le risque de faire fi de la demande du patient...

Mais justement, que demande le patient en 2016? Demande-t-il toujours un travail psychique en profondeur? Est-ce que la demande d' «amputer la part souffrante» (L’Archevêque et Bourgeois-Guérin, 2016) équivaut à amputer le sujet en lui ? Si la demande est d'ainsi se "faire violence ${ }^{6}$ », du moins, de notre point de vue... que peut-on faire? Refuser de le recevoir? Peut-on considérer le patient comme sujet malgré lui, plutôt qu'assujetti à la culture et la société, au regard de l'autre ou encore à l'imaginaire? Le psy se fera-t-il un devoir de délivrer le sujet ou demeurera-t-il témoin silencieux, pétrifié, de cette violence?

La violence est partout, disions-nous. Mais n'est-ce pas là une vérité intemporelle? En effet, du point de vue intrapsychique, on ne saurait abolir la violence, sans amputer justement l'humain, le sujet humain. En ce sens, nous assisterions aujourd'hui essentiellement au retour à l'évidence des fondements de l'humain: violence fondamentale (Bergeret), pulsion de mort, tendance à la décharge pulsionnelle (Freud)... la Nature de l'Homme? À 
l'opposé, du point de vue biologique des sciences de la Nature, l'humain serait en perpétuelle lutte contre les lois du chacun pour soi, du plus fort, de la sélection naturelle. Contre nature, le «sujet» avec son éthique, le sens à trouver voire à construire, son désir de communion voire de communauté, l'importance accordée à l'altérité, la liberté et la dignité, questionnera Ameisen (2007)? Si le malaise dans la culture freudien reflète cette tension entre les lois du biologique et celles de la culture et de la vie en société, au risque que la vie du névrotique s'apparente à une vie contre-nature, reste que le «travail du négatif» (Green) témoigne du potentiel symbolisant, structurant de cette opposition à la pulsion. De fait, l'incontournable potentiel destructeur humain a des vertus évolutives nous dévoilait Winnicott: détruire pour reconstruire, détruire pour se construire en interaction avec l'autre, pour se délier, au moins minimalement et paradoxalement pour créer l'objet et le sujet. De façon générale, la violence demeure la voie privilégiée de la construction, du rapport à l'autre, et du sens, tel que le soutient notamment le concept de castration symboligène (Dolto) inhérente à la subjectivation, à l'autonomisation. Quelle violence doit ainsi être assumée et tolérée par le clinicien pour qu'évolution, voire construction du sujet puisse advenir?

Si le psy a une position privilégiée, c'est donc non seulement par l'inévitable confrontation à la violence de l'autre, mais bien, dans une perspective psychanalytique, par sa référence à une théorie qui propose des voies de compréhension de cette violence ${ }^{7}$. Recréer l'espace pour jouer, pour élaborer, pour représenter et symboliser; tel est le mandat du clinicien d'orientation psychanalytique. La double valence de la violence ne peut, du point de vue psychanalytique, être niée: violence pour vivre ou pour mourir ${ }^{8}$ ?

2016. La violence est partout. Revenons, en conclusion, à ces jeunes qui repoussent les limites de la violence... pour donner un sens - une direction et une signification - à une vie insensée, en entrevoyant la lumière: un Idéal déguisé en Dieu, Mohamed... peu importe. Pour eux, il s'agit de défendre le semblable (l'autre imaginaire) et l'Idéal (l'idéologie, dans les faits, d'où le bémol que l'on peut accorder à la valence symbolique de celuici) en détruisant l'altérité (l'autre différent). Et curieusement, il semble que la réponse qu'ils provoquent se pose d'emblée dans les mêmes termes. Une rapide référence à la psychanalyse ne peut que nous pister vers le danger que constitue une telle identification massive à La Cause assortie de la négation du désir propre (et donc, de l'essence du sujet humain), dans un combat sans merci qui oppose deux clans prisonniers d'une dynamique spéculaire. En ces temps incertains, la psychanalyse n'a-t-elle pas l'obligation d'éclairer 
ces débats sociaux? De donner un sens, une nouvelle voie de compréhension à ce qui apparaît comme l'un des principaux enjeux sociétaux de notre époque? Redonner sens à la vie, autoriser le travail de subjectivation, dans toute sa complexité et sa temporalité, serait-ce là la tâche la plus spécifique des cliniciens d'orientation psychanalytique en 2016? Lourde tâche, n'est-il pas?

\author{
Sophie Gilbert \\ gilbert.sophie@uqam.ca
}

\title{
Notes
}

1. Intéressant de noter la double définition: au-delà de l'allusion à la propreté, la connotation en lien avec la propriété amène à se demander comment cet espace à soi peut-il devenir un espace partagé où le patient pourra créer... à partir de lui-même et non seulement de l'autre?

2. Pensons ici aux messages de propagande associés aux vidéos de mises à mort, ou encore, au message à (re) construire lors de procès alors que l'agir est depuis longtemps «en ligne» ou surmédiatisé - le «cas» Magnotta, par exemple... Ces images sans parole nous semblent à rapprocher du silence des ados qui se présentent en clinique (ils sont tout de même au rendez-vous; ils s'imposent à nous d'une façon provocante, telles ces images surdiffusées dans les réseaux sociaux) et donnent à voir plutôt qu'à entendre.

3. On oublie parfois qu'étymologiquement, ce mot fait allusion à l'étranger.

4. À noter que Ciccone (2014) a mis l'accent récemment sur de telles taches aveugles, dans un collectif intitulé La violence dans le soin.

5. Voir les derniers numéros de Filigrane sur le devenir de la psychanalyse.

6. Ici, le parallèle avec la chirurgie esthétique s'impose: inscrite dans un désir trop souvent mal délimité, ou du moins, mal élaboré avec tout ce qu'on sait de l'éternel recommencement de ces opérations sur le corps qui jamais ne satisfont le sujet. Le corps attaqué par soi: s'agit-il de violence, de pure esthétique, ou même d'un statement? Être assujetti aux standards, ou plutôt à l'imaginaire de son époque?

7. À la lumière de l'éthique (Lacan), l'économique (Freud), du cadre (Roussillon), du discours (Aulagnier), du «mal» (Green), de l'aire de jeu (Winnicott), etc

8. Comme en témoigne d'ailleurs l'étymologie...

\section{Références}

Ameisen, J.-C. (2007). La mort au cœur du vivant, Revue française de psychosomatique, 32 (2), 11-44.

Ciccone, A. (dir.) (2014). La violence dans le soin. Paris: Dunod.

L'Archevêque, A., et Bourgeois-Guérin, É., 2016, Une clinique sans sujet? Chirurgie psychique à l'époque de l'esprit matière. Filigrane, 25 (1).

Lévy, G. (2013). Pour vivre, mourir ou de la tyrannie de l'actuel, conférence donnée le 28 septembre 2013, inédit.

Paillé, P. (2006). Lumières et flammes autour de ma petite histoire de la recherche qualitative, Recherches qualitatives, 26 (1), 139-153. 